\title{
Two-wavelength double heterodyne interferometry using a matched grating technique
}

\author{
Zoran Sodnik, Edgar Fischer, Thomas Ittner, and Hans J. Tiziani
}

\begin{abstract}
Two-wavelength double heterodyne interferometry is applied for topographic measurements on optically rough target surfaces. A two-wavelength $\mathrm{He}-\mathrm{Ne}$ laser and a matched grating technique are used to improve system stability and to simplify heterodyne frequency generation.
\end{abstract}

\section{Introduction}

If an optically rough surface is illuminated by coherent light, the well-known speckle phenomenon occurs. Traditional interferometry on optically rough surfaces becomes difficult or impossible. To circumvent the speckle problem one can increase the wavelength (e.g., $\mathrm{CO}_{2}$ laser) as described by Kwon et al. ${ }^{1}$ Furthermore, a grazing incidence interferometer as shown by Murty and Shukla ${ }^{2}$ can be used. Alternatively two wavelengths can be utilized simultaneously. The sensitivity is reduced to an effective wavelength $\lambda_{\text {ef }}$ given by $\lambda_{\text {ef }}$ $=\lambda_{1} \lambda_{2} /\left|\lambda_{1}-\lambda_{2}\right|$ as shown by Wyant ${ }^{3}$ for a holographic setup and for real time application where an electrooptical crystal (BSO) was used to store the hologram instead of a photographic plate by Kuchel and Tiziani. ${ }^{4}$ Polhemus ${ }^{5}$ demonstrated contrast modulation of interference fringes by $\lambda_{\text {ef }}$ generated with two wavelengths simultaneously. A scanning two-wavelength heterodyne speckle interferometer using a krypton laser was described by Fercher et al. ${ }^{6}$ where two phasemeters are needed to finally calculate the phase of the effective wavelength.

In this paper we describe a scanning double heterodyne technique (two laser wavelengths and heterodyne frequencies are used simultaneously) where a low frequency detection signal with a phase shift that corresponds to the effective wavelength is generated. A two-wavelength double heterodyne interferometer (DHI) setup consists basically of two independent heterodyne interferometers working at different wave-

The authors are with Stuttgart University, Institute for Technical Optics, D-7000 Stuttgart 80, Germany.

Received 23 April 1990.

0003-6935/91/223139-06\$05.00/0.

(C) 1991 Optical Society of America. lengths $\lambda_{1}$ and $\lambda_{2}$ and different heterodyne frequencies $f_{1}$ and $f_{2}$. The phase of the beat frequency $f_{1}-f_{2}$ depends on the effective wavelength $\lambda_{\text {ef }}$ and can therefore be examined for distance evaluation as shown by Dandliker et al. ${ }^{7}$ Using two (highly stable) laser sources emitting different wavelengths, the heterodyne frequency shifts can easily be obtained by acoustooptical modulation (AOM) as shown in Fig. 1. The unshifted wavelengths are combined in a beam splitter (BS) and a monomode fiber (MMF) to generate an identical wavefront before being focused onto an optically rough specimen.

Only identical wavefronts of $\lambda_{1}$ and $\lambda_{2}$ will generate identical speckles in the entrance pupil of the imaging system and onto the detector. After passing a lens (L), a polarizing beam splitter (PBS) and a quarterwave plate the light is focused onto the specimen by a microscope objective (MO) as shown in Fig. 1. The quarterwave plate is passed twice and used to rotate the polarization by $90^{\circ}$ to achieve reflection at the polarizing beam splitter. To match the polarizations of the reference and the target beam the polarization in the target beam is then rotated back by a halfwave plate (HWP) and combined in a beam splitter with the heterodyne frequency shifted beams. The interference signal is observed by a photodetector (Det). The beat frequency is generated by squaring the signals with a Schottky diode and then fed to a phase detector. The reference signal for the phase detector is generated in an additional interference arrangement to compensate for phase fluctuations of the two highly stable laser sources.

In the case of a multiline laser, to be discussed here, the wavelengths $\lambda_{1}$ and $\lambda_{2}$ are perfectly combined and have a good relative stability. In a conventional setup (similar to the one shown in Fig. 1, but with a multiline laser instead of two lasers) the laser radiation would be first divided into target and reference beams. The two wavelengths in the reference beam would be split (e.g., 


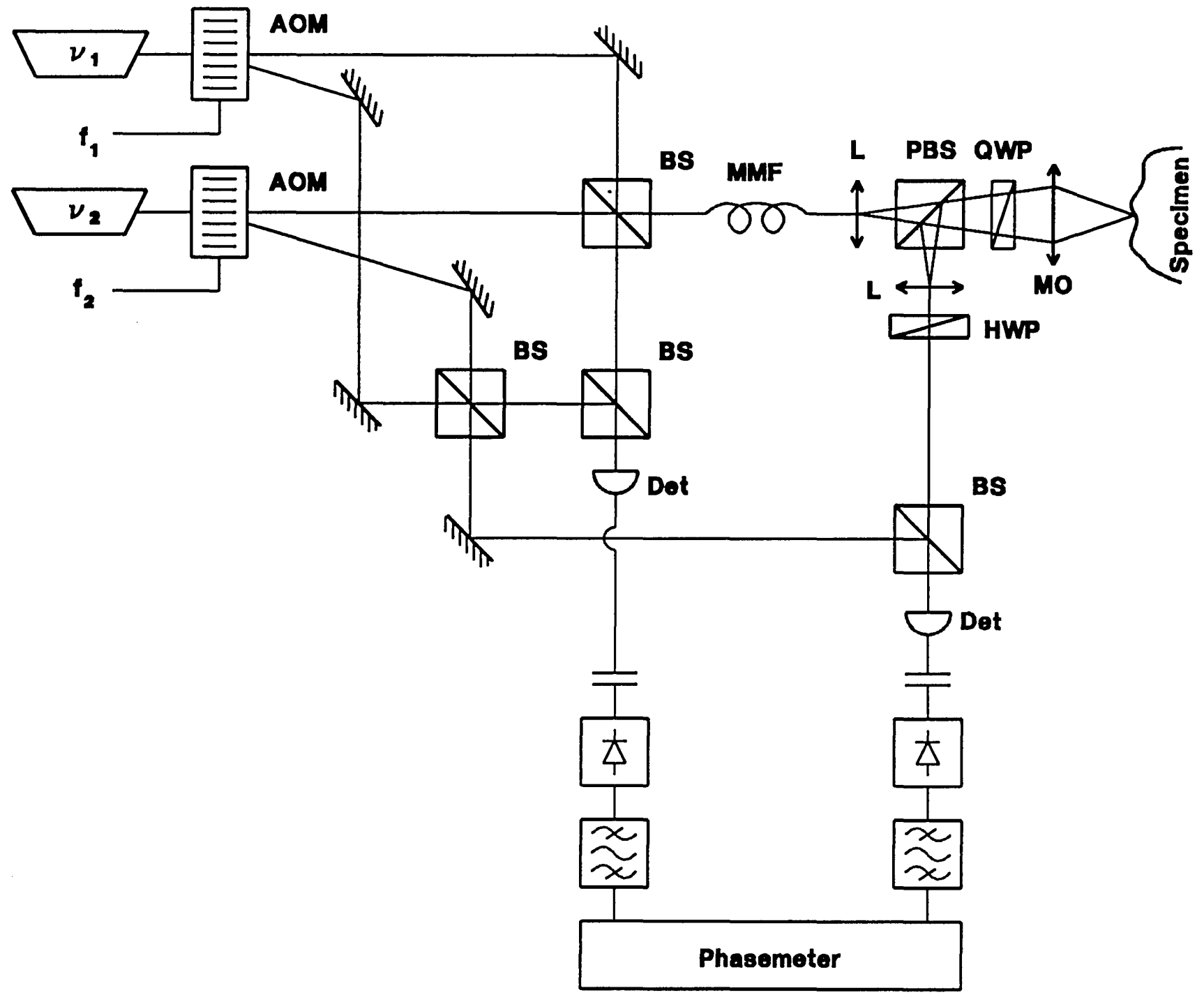

Fig. 1. Schematic double heterodyne interferometry setup.

by first-order diffraction of a grating), frequency shifted (AOM) by $f_{1}$ and $f_{2}$, respectively, and recombined in a beam splitter. Such an approach however would be very sensitive to several sorts of disturbances.

In this paper a matched grating technique is described that minimizes disturbances and increases efficiency. Two examples (which are both using a twowavelength $\mathrm{He}-\mathrm{Ne}$ laser) are given. The principle can be applied to all laser sources that emit two wavelengths simultaneously (e.g., argon laser) and where the wavelength differences are large enough to be separated by a diffraction grating.

\section{Theory of Operation}

Looking at the detector (shown in Fig. 1), the four complex amplitudes are given by

$$
\begin{aligned}
& a_{1}=A_{1} \exp \left[i\left(2 \pi v_{1} t-4 \pi \frac{v_{1}}{c} z\right)\right], \\
& a_{2}=A_{2} \exp \left[i\left(2 \pi v_{2} t-4 \pi \frac{v_{2}}{c} z\right)\right],
\end{aligned}
$$

$$
\begin{aligned}
& a_{3}=A_{3} \exp \left[i\left(2 \pi\left(v_{1}+f_{1}\right) t-2 \pi \frac{v_{1}+f_{1}}{c} l_{1}\right)\right], \\
& a_{4}=A_{4} \exp \left[i\left(2 \pi\left(v_{2}+f_{2}\right) t-2 \pi \frac{v_{2}+f_{2}}{c} l_{2}\right)\right] .
\end{aligned}
$$

Equations (1) and (2) describe the target path and Eqs. (3) and (4) the reference path. The terms $A_{1}, A_{2}$, $A_{3}$, and $A_{4}$ indicate the individual amplitudes, $v_{1}$ and $v_{2}$ are the two laser frequencies, $c$ is the speed of light, $f_{1}$ and $f_{2}$ are the heterodyne frequencies, and $l_{1}$ and $l_{2}$ are the individual reference path lengths. It is assumed that in $l_{1}$ and $l_{2}$ all possible interferometer pathlengths (not the target path $z$ ) are included. The intensity $I$ at the detector is given by

$$
\begin{aligned}
I=\left|a_{1}+a_{3}\right|^{2}+\left|a_{2}+a_{4}\right|^{2} & \\
& =\left(a_{1}+a_{3}\right)\left(a_{1}^{*}+a_{3}^{*}\right)+\left(a_{2}+a_{4}\right)\left(a_{2}^{*}+a_{4}^{*}\right) .
\end{aligned}
$$

The asterisk indicates complex conjugate terms. Inserting Eqs. (1), (2), (3), and (4) in Eq. (5) leads to 


$$
\begin{aligned}
& I=A_{1}^{2}+ A_{2}^{2}+A_{3}^{2}+A_{4}^{2} \\
&+2 A_{1} A_{3} \cos \left(2 \pi f_{1} t+4 \pi \frac{v_{1}}{c} z-2 \pi \frac{v_{1}+f_{1}}{c} l_{1}\right) \\
& \quad+2 A_{2} A_{4} \cos \left(2 \pi f_{2} t+4 \pi \frac{v_{2}}{c} z-2 \pi \frac{v_{2}+f_{2}}{c} l_{2}\right) .
\end{aligned}
$$

The detector must be fast enough to detect the heterodyne frequencies $f_{1}$ and $f_{2}$. The diopter term is removed from the detector signal by a capacitor. None of the terms in Eq. (6) is dependent on the effective wavelength $\lambda_{\text {ef }}$. It is obtained by squaring the detector signal. This is approximately achieved by applying it to a nonlinear element (e.g., diode). Cutting off the diopter intensity and squaring the terms given in Eq. (6) leads to

$$
\begin{aligned}
I^{2}= & 2 A_{1}^{2} A_{3}^{2}+2 A_{2}^{2} A_{4}^{2} \\
& +2 A_{1}^{2} A_{3}^{2} \cos \left(4 \pi f_{1} t+8 \pi \frac{v_{1}}{c} z-4 \pi \frac{v_{1}+f_{1}}{c} l_{1}\right) \\
& +2 A_{2}^{2} A_{4}^{2} \cos \left(4 \pi f_{2} t+8 \pi \frac{v_{2}}{c} z-4 \pi \frac{v_{2}+f_{2}}{c} l_{2}\right) \\
& +4 A_{1} A_{2} A_{3} A_{4} \cos \left(2 \pi\left(f_{1}-f_{2}\right) t+4 \pi \frac{v_{1}-v_{2}}{c} z-2 \pi \frac{v_{1}+f_{1}}{c} l_{1}\right. \\
& \left.+2 \pi \frac{v_{2}+f_{2}}{c} l_{2}\right) \\
& 4 A_{1} A_{2} A_{3} A_{4} \cos \left(2 \pi\left(f_{1}+f_{2}\right) t+4 \pi \frac{v_{1}+v_{2}}{c} z-2 \pi \frac{v_{1}+f_{1}}{c} l_{1}\right. \\
& \left.-2 \pi \frac{v_{2}+f_{2}}{c} l_{2}\right)
\end{aligned}
$$

The phase of the lowest frequency term $\left(f_{1}-f_{2}\right)$ in Eq. (7) now corresponds to the wanted effective wavelength $\lambda_{\text {ef }}=c /\left(v_{1}-v_{2}\right)$. From the frequency spectrum in Eq. (7) this term is bandpass filtered. The result is a low frequency signal $\left(f_{1}-f_{2}\right)$ with a phase that depends on the target distance $z$. The distance sensitivity is reduced to the effective wavelength.

\section{Discussion of Error Sources}

\section{A. Inherent DHI Error Sources}

An analysis of the low frequency term $\left(f_{1}-f_{2}\right)$ in Eq. (7) shows that several components in the interferometer setup affect the accuracy of the phase (distance) measurement. Errors from fluctuations of the heterodyne frequencies $f_{1}$ and $f_{2}$ are small. These fluctuations alter the beat (detection) frequency, but do not affect the phase measurement because the heterodyne frequencies are by seven orders of magnitude lower than the laser frequencies. Nevertheless stabilization of $f_{1}$ and $f_{2}$ is required because the electronic signal processing needs bandpass filtering. If the heterodyne frequencies vary, phase errors are introduced by a bandpass filter as well as by a phasemeter.

The phase response $\phi$ in the relevant term of Eq. (7) is summarized to

$$
\phi=4 \pi\left(\frac{v_{1}-v_{2}}{c}\right) z-2 \pi\left(\frac{v_{1} l_{1}-v_{2} l_{2}}{c}\right),
$$

where the heterodyne frequencies $f_{1}$ and $f_{2}$ are omitted for reasons mentioned above. For a phase stability analysis the derivative of Eq. (8) is given by

$$
\begin{aligned}
& \delta \phi= \frac{\partial \phi}{\partial v_{1}} \delta v_{1}+\frac{\partial \phi}{\partial v_{2}} \delta v_{2}+\frac{\partial \phi}{\partial l_{1}} \delta l_{1}+\frac{\partial \phi}{\partial l_{2}} \delta l_{2}, \\
& \delta \phi=\left(4 \pi \frac{z}{c}-2 \pi \frac{l_{1}}{c}\right) \delta v_{1}+\left(-4 \pi \frac{z}{c}+2 \pi \frac{l_{2}}{c}\right) \delta v_{2} \\
&-2 \pi \frac{v_{1}}{c} \delta l_{1}+2 \pi \frac{v_{2}}{c} \delta l_{2} .
\end{aligned}
$$

Equation (9) shows that, for accurate distance measurements, laser frequency fluctuations $\delta v_{1}=\delta v_{2}=\delta v$ as well as reference pathlength fluctuations $\delta l_{1}=\delta l_{2}=$ $\delta l$ should be identical. Both requirements are difficult to achieve in a two laser setup (as shown in Fig. 1), but can be fulfilled with a matched grating technique combined with a single multiline laser that generates two wavelengths simultaneously.

A matched grating technique was developed to minimize the separation of the interferometer reference beams. Consequently, we found that if the polarization properties of both laser wavelengths are not identical, birefringence makes the requirement $\delta l_{1}=\delta l_{2}$ very hard to fulfill. With this in mind Eq. (9) can be simplified to

$$
\delta \phi=\frac{2 \pi}{c} \Delta l \delta v+\frac{2 \pi}{\lambda_{\mathrm{ef}}} \delta l,
$$

with the reference pathlengths difference $\Delta l=l_{1}-l_{2}$ and the effective wavelength $\lambda_{\text {ef }}=c /\left(v_{1}-v_{2}\right)$. Our experimental arrangements (shown in Figs. 2 and 3 ) were almost phase insensitive $\left(\delta \phi<0.1^{\circ}\right)$ to laser frequency fluctuations $\delta v$ because of a reference path difference $\Delta l$ of $<1 \mathrm{~mm}$. Finally, the sensitivity to pathlength fluctuations in the reference beam $\delta l$ (as well as in the target beam) is related to the effective wavelength $\lambda_{\text {ef. }}$.

\section{B. Error Sources from Rough Surfaces and Defocus}

Although a DHI virtually works with an effective (long) wavelength $\lambda_{\text {ef }}$, the target is illuminated by two short laser wavelengths $\lambda_{1}$ and $\lambda_{2}$ that generate speckles on an optically rough surface. The influence of varying speckle intensities on phase error probabilities was theoretically and experimentally examined by Fercher et al. ${ }^{6}$ We found their results confirmed, but in our measurements the target distances were small and strong intensities occurred. By the choice of the integration time of the phase meter we were able to compensate for phase errors caused by temporary signal fading.

A second problem is the system's sensitivity to defocus. It is related to the laser wavelengths $\lambda_{1}$ and $\lambda_{2}$, because interference of target and reference beams occurs before an effective wavelength $\lambda_{\text {ef }}$ is (electronically) obtained. Spherical interference fringes occur on the photodetector and decrease the modulation contrast. 


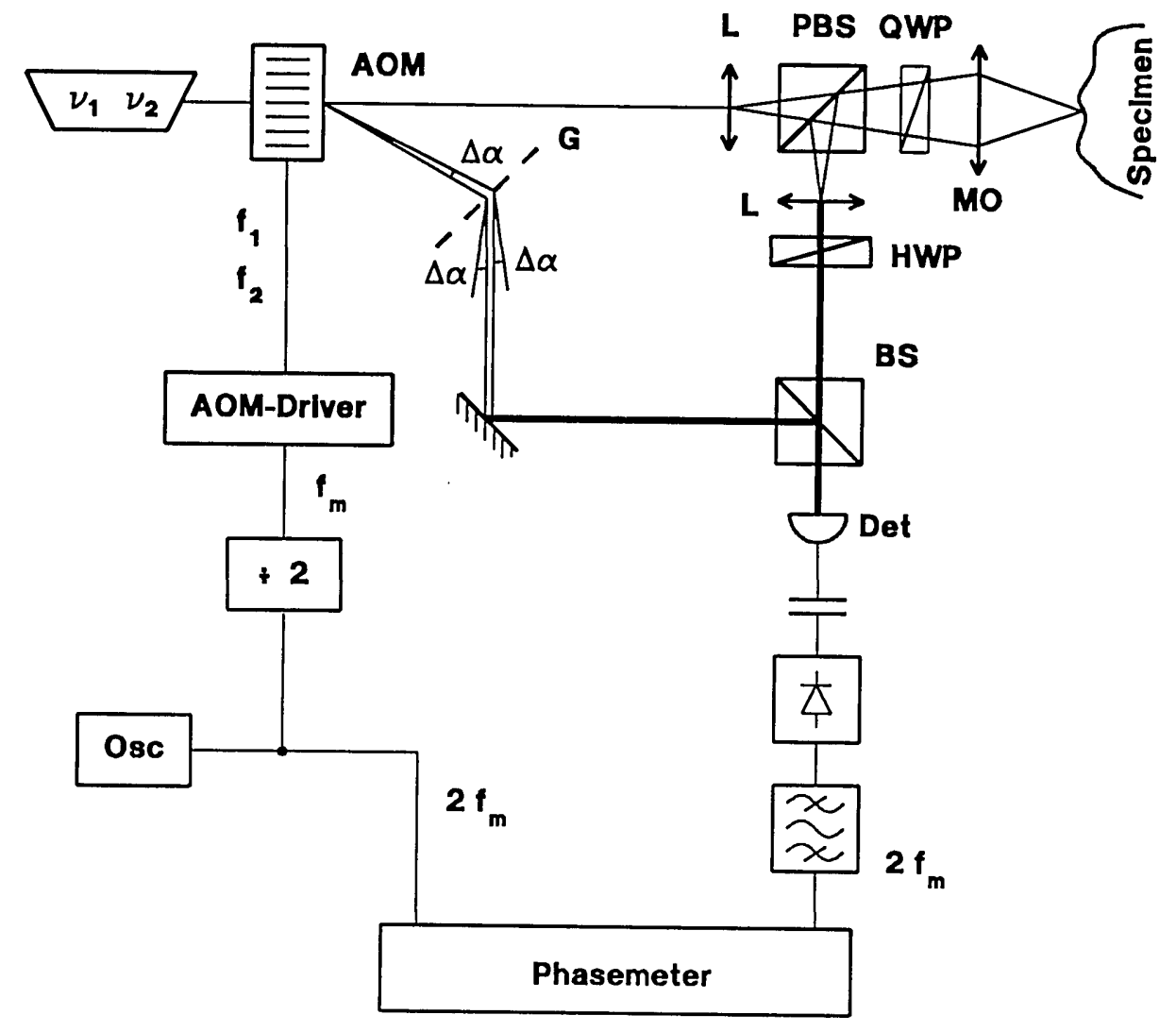

Fig. 2. Double heterodyne interferometer with solid-state matched gratings.

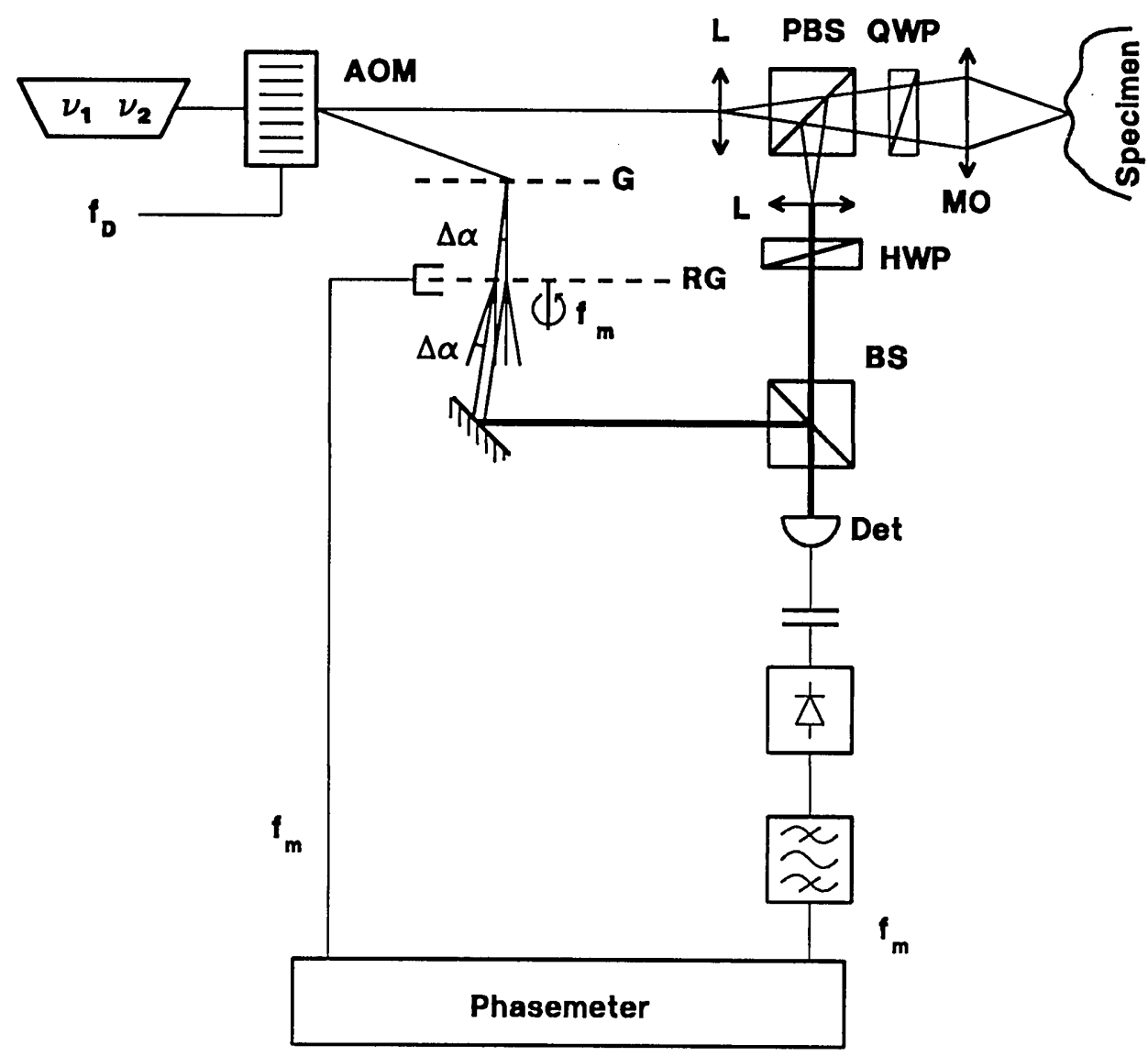

Fig. 3. Double heterodyne interferometer with a rotational matched grating. 


\section{Experiments}

A. Double Heterodyne Interferometer with Solid State Matched Gratings

Figure 2 shows a multiwavelength $\mathrm{He}-\mathrm{Ne}$ laser (Spindle \& Hoyer) tuned to emit simultaneously and with equal intensity at $632.8 \mathrm{~nm}$ and $640.1 \mathrm{~nm}$. This leads to an effective wavelength of $55.5 \mu \mathrm{m}$. As shown, only one AOM is required (compared to the setup shown in Fig. 1). The AOM is operated in suppressed carrier mode, where a modulation signal $f_{m}$ is applied to the AOM driver, while the driver carried amplitude is turned to zero. This results in a multiplication of the driver carrier frequency $f_{d}$ with the modulation frequency $f_{m}$ leading to two side bands $\left(f_{1}=f_{d}+f_{m}\right.$ and $f_{2}=f_{d}-f_{m}$ ) and suppressed carrier $f_{d}$. As a result, the AOM is operated at two frequencies, $f_{1}$ and $f_{2}$, simultaneously. Two individual phase gratings are established in the AOM crystal leading to two first-order beams that leave the AOM under slightly different angles $\alpha_{1}$ and $\alpha_{2}$. The diffraction efficiency (the energy distribution between the zero-order and the two first-orders) is optimized (Bragg angle) and adjusted with the driving power of the modulation signal $f_{m}$. In this way the frequencies of the two first-order diffracted beams are $v_{1}+f_{d}-f_{m}, v_{2}+f_{d}-f_{m}$ and $v_{1}+f_{d}+f_{m}$, respectively. The small angle $\Delta \alpha=\alpha_{1}-\alpha_{2}$ between the two first-order diffracted beams is approximately given by

$$
\Delta \alpha=2 \frac{\lambda}{v} f_{m}
$$

where $\lambda=\lambda_{1}$ or $\lambda_{2}$, one of the $\mathrm{He}-\mathrm{Ne}$ laser wavelengths (the spatial frequency of the AOM phase grating is far too low to separate the two laser wavelengths $\lambda_{1}$ and $\lambda_{2}$ ). The term $v$ is the speed of sound in the AOM crystal.

In our new approach, we matched the angle $\Delta \alpha$ to the difference of the first-order diffraction angle (between $\lambda_{1}$ and $\lambda_{2}$ ) of a high spatial frequency grating $(G)$.

The small angle $\Delta_{\alpha}$ between the first-order diffraction of $\lambda_{1}$ and $\lambda_{2}$, respectively, is approximately given by

$$
\Delta \alpha=\lambda_{2} g \sqrt{1-\left(\lambda_{1} g\right)^{2}}-\lambda_{1} g \sqrt{1-\left(\lambda_{2} g\right)^{2}},
$$

with the spatial frequency $g$ of the grating. Matching two gratings accordingly, only beams with frequencies $v_{2}+f_{d}-f_{m}$ and $v_{1}+f_{d}+f_{m}$ become parallel. They are extracted to serve as reference for the interferometer as shown in Fig. 2. In this setup there is no (or hardly any) reference pathlength difference between $l_{1}$ and $l_{2}$ introduced as compared to the setup shown in Fig. 1. This makes the system almost insensitive to laser frequency variations. The shear of the reference beams shown in Fig. 2 is negligible because of a very small divergence angle $\Delta \alpha$. The modulation frequency is generated by an oscillator and divided by two before being applied to the AOM driver. Because of the two sidebands generated, the beat frequency is twice that of the modulation frequency $f_{m}$. Further downmixing of the signals is helpful, since highly accurate phase meters are easier to obtain that work at low frequencies.

\section{B. Double Heterodyne Interfeometer with a Rotational Matched Grating}

In the setup shown in Fig. 3, the AOM is operated conventionally at driver frequency $f_{d}$. This shifts both $\mathrm{He}-\mathrm{Ne}$ laser frequencies in the beam that leaves the AOM in Bragg diffraction. The two frequencies $v_{1}+f_{d}$ and $v_{2}+f_{d}$ are (first-order) diffracted a second time with a high spatial frequency $(600 \mathrm{lp} / \mathrm{mm})$ grating at angles $\alpha_{1}$ and $\alpha_{2}$, respectively. The grating is calculated (and produced on photoresist) in a way that the diffraction angle difference $\Delta \alpha$ between $v_{1}+f_{d}$ and $v_{2}+$ $f_{d}$ is the same as the first-order diffraction angle of a following (low spatial frequency) grating for the average $\mathrm{He}-\mathrm{Ne}$ wavelength $\lambda=\lambda_{1}$ or $\lambda_{2}$ (considering $\lambda_{1}$ and $\lambda_{2}$ to be nearly the same for a low spatial frequency grating).

In this way the first-order diffraction of $v_{1}+f_{d}$ and the zero-order of $v_{2}+f_{d}$, as well as the zero-order of $v_{1}+$ $f_{d}$ and the minus first-order of $v_{2}+f_{d}$, become parallel as shown in Fig. 3. The second grating (RG) is a standard low cost angle encoder wheel (Hewlett Packard) with a spatial frequency of $7.2 \mathrm{lp} / \mathrm{mm}$. It is continuously rotated by a motor. Because of rotation of the grating, the first-order diffracted light is frequency shifted by $f_{m}$ while the zero-order light passes the grating unaffected. Selecting one pair of paralleled beams (as shown in Fig. 3) the frequencies of the beams are $v_{1}+f_{d}+f_{m}=v_{1}+f_{1}$ and $v_{2}+f_{d}=v_{2}+f_{2}$. They serve as reference for the interferometer. The beat frequency $f_{m}=f_{1}-f_{2}$ is generated, after interference of reference and target beam, by rotation of the angle encoder. In our experiment the angle encoder wheel had $1000 \mathrm{lp} /$ revolution and was rotated by $1200 \mathrm{rpm}$, therefore a heterodyne beat frequency of $20 \mathrm{KHz}$ was found to be most appropriate. This frequency can be directly applied to an accurate low frequency phase meter (e.g., two channel lockin amplifier).

The reference for the lockin amplifier is directly taken from the angle encoder detector (square wave signal) output. Care must be taken, however, in the design of the encoder wheel bearing. During rotation different kinds of mechanical resonances can lead to phase fluctuations in the reference beam. Figure 4 shows the detector signal. The beat frequency $f_{m}=f_{1}$ $-f_{2}$ is shown in the upper part of the oscillogram and the squared and bandpass filtered signal in the lower part.

\section{Results}

The experimental results were obtained with a $10 \times$ microscope objective and an avalanche photodetector. The diffraction limited target spot was of the order of 3 $\mu \mathrm{m}$ and the clear working distance to the target was 6.5 $\mathrm{mm}$. The unoptimized measurement speed of about ten samples per second could be considerably increased due to availability of a strong target return signal. We calculated the SNR to be $45 \mathrm{~dB}$ in the 


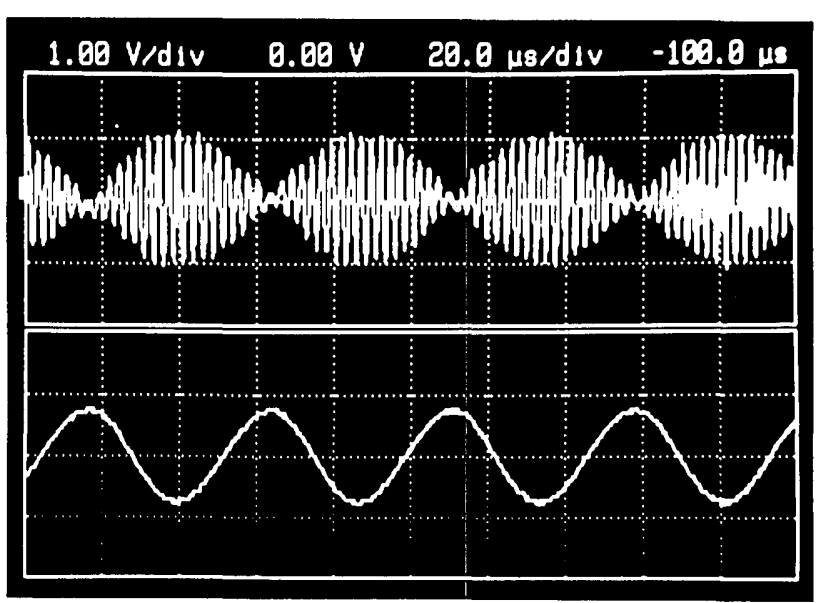

Fig. 4. (Upper part) superposition of heterodyne frequencies; (lower part) squared and band-pass filtered heterodyne beat signal.

presence of a bright speckle. The target was moved with a computer controlled stepper motor.

Figure 5 shows the phase response in a measurement where the target distance to a mirror was varied by 100 $\mu \mathrm{m}$. The unambiguous distance range $\left(\lambda_{\text {ef }} / 2\right)$ of 27.75 $\mu \mathrm{m}$ as well as the good linearity are shown. Two successive topography measurements on a machined aluminum sample are shown in Fig. 6. On the sample, two steps of 5 and $10 \mu \mathrm{m}$ were milled into the surface. The topography is superimposed by a periodic height variation of the scanning table. It corresponds to one revolution (this is a scan of $0.5 \mathrm{~mm}$ ) of the driving motor. In the first provisional experimental setup a resolution of the order of $0.5 \mu \mathrm{m}$ was obtained. Some occasional height differences in both measurements can be explained by slow thermal expansion of the motor and mechanical vibration of the rotational grating. It is expected that the accuracy and the resolution can be increased considerably by the use of a solid state approach where an AOM is used instead of a rotational grating as mentioned above. These results will be presented in a later paper.

\section{Conclusions}

Two-wavelength double heterodyne interferometry proved to be a powerful tool for sensitivity reduced interferometric measurements on smooth as well as on optically rough surfaces. We combined a dual wavelength $\mathrm{He}-\mathrm{Ne}$ laser (632.8 and $640.1 \mathrm{~nm}$ ) with a matched grating technique to prevent some inherent DHI problems and to simplify the setup. The unambiguous distance range of the DHI was $27.75 \mu \mathrm{m}$ and enabled measurements on machined aluminum samples. Intensity thresholding to reject phase (distance) data of signals with low amplitudes, as suggested by Fercher et al., ${ }^{6}$ was not required in our experiments. The strong signals involved allowed us to compensate for signal fading and related phase errors by appropriate choice of phasemeter integration time while scanning. The matched grating principle could be used with a different optical projection system to build a

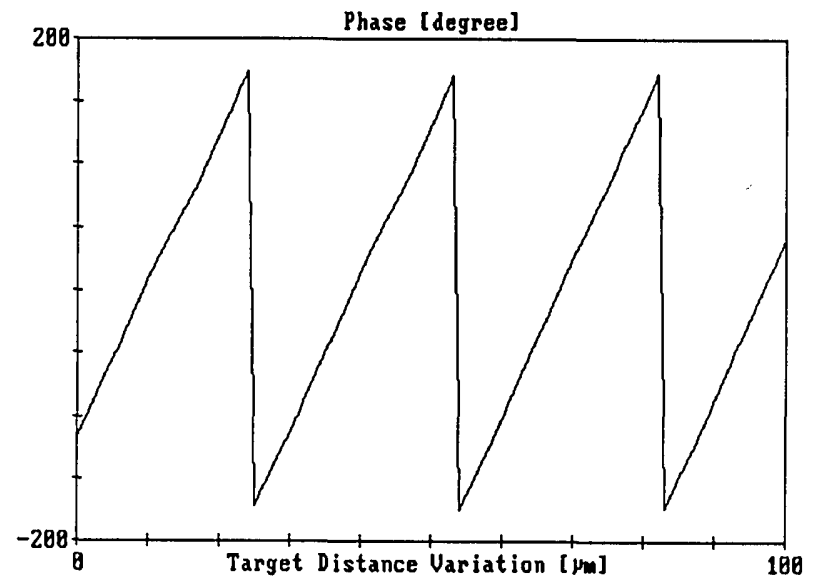

Fig. 5. Phase response from a target distance variation of $100 \mu \mathrm{m}$.

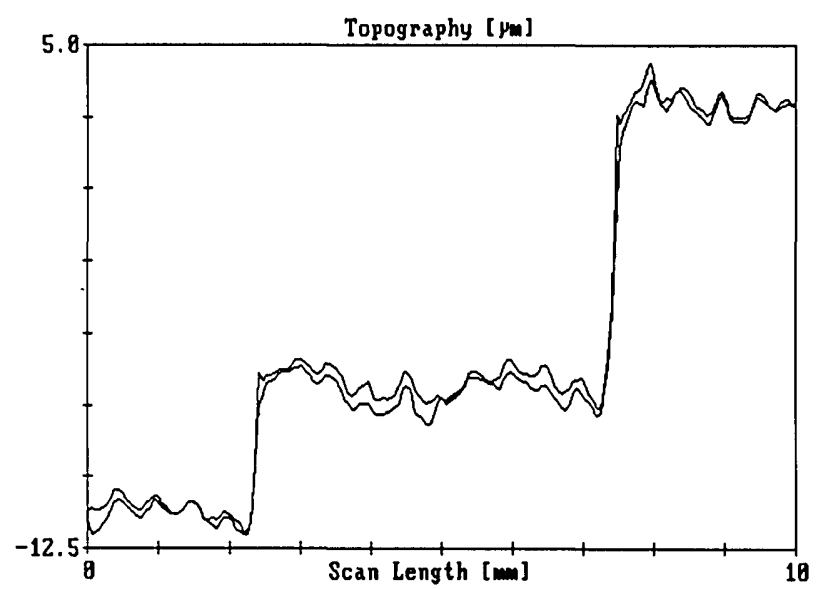

Fig. 6. Topography obtained from two successive line scans on a milled aluminum sample with step heights of $5 \mu \mathrm{m}$ and $10 \mu \mathrm{m}$ and superimposed by periodic height variations from the scan table.

highly accurate metrological instrument for distances up to several meters.

The authors like to thank S. Manhart and R. Maurer from Messerschmitt Bolkow-Blohm, A. Mariani, C. Giunti, R. Bonsignori, and S. Zatti from Officine Galileo and P. Roussel from the European Space and Technology Center for the support and fruitful discussions.

\section{References}

1. O. Kwon, J. C. Wyant, and C. R. Hayslett, "Rough Surface Interferometry at $10.6 \mu \mathrm{m}$," Appl. Opt. 19, 1862-1869 (1980).

2. M. V. R. K. Murty and R. P. Shukla, "An Oblique Incidence Interferometer," Opt. Eng. 15, 451 (1976).

3. J. C. Wyant, "Testing Aspherics Using Two-Wavelength Holography," Appl. Opt. 10, 2113-2118 (1971).

4. F. M. Kuchel, and H. J. Tiziani, "Real-Time Contour Holography Using BSO Crystals," Opt. Commun. 38, 17 (1981).

5. C. Polhemus, "Two-Wavelength Interferometry," Appl. Opt. 12, 2071-2074 (1973).

6. A. F. Fercher, H. Z. Hu, and U. Vry, "Rough Surface Interferometry with a Two-Wavelength Heterodyne Speckle Interferometer," Appl. Opt. 24, 2181-2288 (1985).

7. R. Dandliker, R. Thalmann, and D. Prongue, "Two-Wavelength Laser Interferometry Using Super-Heterodyne Detection," Proc. Soc. Photo-Opt. Instrum. Eng. 813 (1987). 